\title{
Severe COVD-19 Cases: Is Respiratory Distress Partially Explained by Central Nervous System Involvement?
}

\author{
Calixto Machado-Curbelo MD PhD DSc FAAN
}

The main characteristics and challenging symptoms of COVID-19, caused by the novel coronavirus SARS-CoV-2, are related to respiratory distress. Although most patients have mild symptoms such as fever, headache, cough, myalgia and anosmia, some develop acute respiratory distress syndrome, leading to death in many cases.

Human coronavirus (CoVs) were responsible for two previous worldwide outbreaks: Severe Acute Respiratory Syndrome (SARS-CoV) and Middle East Respiratory Syndrome (MERSCoV). Several reports of these outbreaks demonstrated that these diseases affected the central nervous system (CNS).[1] Thus, for the current COVID-19 pandemic, a crucial question arises: does CNS affection at least partially explain the respiratory distress commonly found in these patients?

SARS-CoV-2 is a beta-coronavirus sharing similarities with SARS-CoV. Thus far, it has been postulated that the virus uses glycoproteins expressed on its surface to bind the receptor of the angiotensin-converting enzyme 2 (ACE2), which is distributed in respiratory tract epithelial cells, lung parenchyma and other areas such as the gastrointestinal tract and endothelial cells. The brain expresses ACE2 receptors as well. Those receptors have been detected over glial cells and neurons, both in the cortex and brainstem; therefore, these cells are targets of SARS-CoV-2.[1,2]

Extra-pulmonary symptoms have been described in these viruses, highlighting their capacity to cause neurological complications: febrile seizures, loss of consciousness, convulsions, ataxia, status epilepticus, encephalitis, myelitis, neuritis and Guillain-Barré syndrome, both in humans and experimental models. Preliminary data suggest that sudden olfactory deprivation happens in about $30 \%$ to $50 \%$ of SARS-CoV-2 infected patients. I have read that anosmia and ageusia (loss of smell and taste) can be initial warning signs or unique symptoms of COVID-19 and have reviewed the most common mechanisms of these signs in this disease.[2]

Several routes used by neurotropic viruses to reach the CNS have been described, including transneural and hematogenous pathways. CoVs have been found to reach the brain via the olfactory bulb, and from there, spread into the CNS and periphery. This pathway is an excellent mechanism to access the CNS for viruses that enter the body intranasally. The olfactory nerve has the peculiarity that it communicates with the nasal epithelium and also with the olfactory bulb, a CNS entryway. Experimental models have demonstrated that olfactory nerve ablation limits viral neurotropic capacities in mice. In humans, as soon as the infection is established, the virus can spread to the whole brain and cerebrospinal fluid in less than seven days. Consequently, it has been reported that these viruses can induce demyelination in studies of neuron and glial cultures.[1]

Some research has found that SARS-CoV-2 affects the brain. A recent study describes neurological manifestations in $36.4 \%$ of
214 COVID-19 patients from Wuhan city, China. These include acute cerebrovascular diseases, consciousness impairment and skeletal muscle symptoms, suggesting a neurotropic potential for SARS-CoV-2.[3]

China's National Health Commission's Diagnosis and Treatment Guidelines for COVID-19 published human histopathological studies finding edema and partial neuronal degeneration in brain tissues. Another study reported presence of SARS-CoV-2 in the cerebrospinal fluid of a COVID-19 patient.[1,2]

Firsthand reports in Wuhan hospitals indicate COVID-19's common symptoms were fever (83\%-99\%) and dry cough $(59 \%-82 \%)$ at the onset of disease. However, the most characteristic and deadly symptom was respiratory distress. Among patients with dyspnea, more than half needed mechanical respiratory support. Some $46 \%-65 \%$ of patients in intensive care units quickly worsened and died due to respiratory failure.[3] Wang reported that $11.1 \%$ received high-flow oxygen therapy, $41.7 \%$ received noninvasive ventilation and $47.2 \%$ received invasive ventilation.[4] These data indicate that most (about 89\%) COVID-19 patients in intensive care units required mechanical ventilation.

CoV infections have been reported in the brain of patients and experimental animals, finding the brainstem to be heavily infected.[1,2] Furthermore, some coronaviruses have demonstrated their capacity to spread via a synapse-connected route to medullary cardiorespiratory centers.

It has been widely supported that such medullar lesions can cause respiratory dysfunction-as in the case of congenital central hypoventilation syndrome-or severe brain injury, which results in damage to medullar respiratory control function. Viral antigens have been detected in the brainstem, where the infected regions included the nucleus of the solitary tract and nucleus ambiguous. Afferent axons from the carotid and aortic bodies in the glossopharyngeal nerve contain chemoreceptor cells, and vagal afferent nerves from receptors in the lung communicate with the medulla and respiratory control centers to coordinate and regulate inspiration and expiration, while the efferent fibers from the nucleus ambiguous and the nucleus of the solitary tract provide innervation to airway smooth muscle, glands and blood vessels. Such afferent and efferent neuroanatomic interconnections indicate that the death of infected animals or patients due to respiratory distress may be caused by dysfunction of the brainstem's respiratory center.[1]

In conclusion: as the COVID-19 pandemic expands, I believe there is an urgent need to understand the neurotropic potential of SARS-CoV-2 in order to prioritize and individualize treatment protocols, and to prompt physician attentiveness to neurological symptoms as soon as they appear. This would assist in making possible earlier diagnosis, and hence earlier actions, such as brain edema treatment. It is also important to note that 
SARS-CoV-2 can invade the brainstem, leading to respiratory center dysfunction.

Hence, serial neurological examinations are recommended, with exhaustive exploration of brainstem reflexes, as soon as the first symptoms appear in COVID-19's clinical course. As neuroimaging techniques (CT-Scan and MRI) are not always easy to use in ventilated patients, ancillary tests (such as brainstem auditory and somatosensory evoked potentials, quantitative EEG and transcranial Doppler) can be used to monitor brain function in severely ill COVID-19 patients.

Acute respiratory distress in such patients may be partially explained by brainstem dysfunction. This suggests the need for more specific and aggressive treatments with direct participation by intensivists and neurologists. $-1 /$ -

1. Machado C, Gutiérrez JV. Brainstem dysfunction in SARS-COV2 infection can be a potential cause of respiratory distress. Preprints [Internet]. 2020 [cited 2020 Apr 20]. 2020040330. DOI:10.20944/preprints202004.0330.v1). Available at: https://www.preprints.org/manuscript/202004.0330/v1

2. Machado C, Gutiérrez JV. Anosmia and ageusia as initial or unique symptoms after SARS-COV-2 virus infection. Preprints [Internet]. 2020 [cited 2020 Apr 20]. 2020040272.DOI:10.20944/preprints202004.0272.v1). Available at: https://www .preprints.org/manuscript/202004.0272/v1

3. Huang C, Wang Y, Li X, Ren L, Zhao J, Hu Y, et al. Clinical features of patients infected with 2019 novel coronavirus in Wuhan, China. Lancet [Internet]. 2020 Feb 15 [cited 2020 Apr 15];395(10223):497-506. Available at: https:// www.thelancet.com/journals/lancet/article/PIIS0140-6736(20)30183-5/fulltext

4. Wang Z, Yang B, Li Q, Wen L, Zhang R. Clinical Features of 69 Cases with Coronavirus Disease 2019 in Wuhan, China. Clin Infect Dis [Internet]. 2020 Mar 16 [cited 2020 Apr 16]. DOI: 10.1093/cid/ciaa272. [Epub ahead of print]. Available at: https://www.unboundmedicine.com/medline/citation/32176772/ Clinical_Features_of_69_Cases_with_Coronavirus_Disease_2019_in_Wu han_China_

Submitted: April 21, 2020

Approved for publication: April 23, 2020

Disclosures: None

Correspondence: braind@infomed.sld.cu 